\title{
Integration of Multiple Data Sources for predicting the Engagement of Students in Practical Activities
}

\author{
Llanos Tobarra ${ }^{1}$, Salvador Ros ${ }^{1}$, Roberto Hernández ${ }^{1}$, Antonio Robles-Gómez ${ }^{1}$, Agustín C. \\ Caminero $^{1}$, and Rafael Pastor ${ }^{1}$ \\ ${ }^{1}$ Communication and Control System Department, from Spanish University for Distance Education \\ (Universidad Nacional de Educación a Distancia, UNED)
}

\begin{abstract}
This work presents the integration of an automatic assessment system for virtual/remote laboratories and the institutional Learning Management System (LMS), in order to analyze the students' progress and their collaborative learning in virtual/remote laboratories. As a result of this integration, it is feasible to extract useful information for the characterization of the students' learning process and detecting the students' engagement with the practical activities of our subjects. From this integration, a dashboard has been created to graphically present to lecturers the analyzed results. Thanks to this, faculty can use the analyzed information in order to guide the learning/teaching process of each student. As an example, a subject focused on the configuration of network services has been chosen to implement our proposal.
\end{abstract}

Keywords - Learning Analytics (LA), Assessment and Evaluation Strategies, Virtual/Remote Laboratories, Collaborative Tools, Distance Education.

\section{INTRODUCTION}

$\mathrm{T}$ HE evaluation procedure is a key element within the process of learning. Basically, it allows faculty to check whether educative objectives are accomplished, not only by students, but also by all the participants involved in an educative program [25], such as pedagogical resources. As a consequence, lecturers are required to adapt the learning process to students' needs or preferences, reinforcing or extending it if necessary, according to the European Higher Education Area (EHEA) [27]. The importance of evaluation procedures is even greater at distance Universities since their students' learning process is different from that of face-to-face universities. In distant Universities, students must be more independent and self-demanding since there are no tight schedules, and this heavily affects the evaluation process. By means of evaluation, faculty can select the suitable learning results and adapt dynamically the subject contents to students [22].

On the other hand, adaptive hypermedia has been widely used for the development of customized Web-based courses in the field of Education [3]. Therefore, the students' learning process was guided, adapting both pedagogical resources and learning ways to specific user's features. Since lecturers adapt course materials to students' skills and usage data dynamically [15], they were able to acquire more knowledge in less time. ELM-ART [31] and TANGOW [4] are some examples of traditional educational adaptive systems. The students' interaction in these types of architectures is different from face-to-face students, as stated in [28]. In particular, students have to be able to adapt their communication way to the user interfaces of systems adapted to the students' needs [14].

It is also important to include collaborative issues taking into account the students' behavior. The most relevant research works related to adaptation in Computer Support for Collaborative Learning (CSCL) systems are COALE [9], WebDL [12], and COL-TANGOW [4]. COALE is a collaborative environment where different exercises are recommended to students. The main goal in WebDL is to facilitate user access to services. It focuses on adaptive support for navigation. COL-TANGOW is also a system that supports the dynamic generation of adaptive Web-based courses by selecting, at every step and for each student, the most suitable activities to be proposed.

Nowadays, the evolution of the Web 2.0 allows us to develop more sophisticated techniques to analyze more efficiently the students' learning process, in order to improve the learning contents and structure of a course. One of the most recent research areas is Learning Analytics (LA) [5], [7], [19] in order to discover and organize the information contained in the educational platform. Its main goal is to discover and organize the existing information in order to extract useful knowledge during the teaching/learning process.

Thus, this work is focused on a case of study in which two sources of information, AutoES (our automatic assessment system for virtual/remote laboratories) and the institutional Learning Management System (LMS), are aggregated to analyze the students' progress and their collaborative learning in virtual/remote laboratories. Guiding this process the following research questions arise:

1. Are the students engaged with the proposed practical activities or are they at risk of quitting the activities? 
2. Can we create a system that helps to evaluate if the proposed activities are well-designed?

Within the context of the evaluation activities, two different learning processes have been detected. First, the practical experimentation in the virtual/remote laboratory with the virtual machines picked up by AutoES and, second, the students' knowledge creation through the discussion threats contained in the evaluation forums. Both of these learning processes are highlighted by lecturers when they are asked about how they perform the evaluation of students. So, there is a strong need to aggregate both data sources in order to answer to the aforementioned questions. In order to present these aggregated data to lecturers, a dashboard has been developed. This dashboard contains quantitative and qualitative information for lecturers about the students' experimental and collaborative progress during the evaluation procedure. These data will be validated by means of a set of learning indicators and their graphical visualization. In particular, the dashboard shows a set of evaluation events for each activity, the students' social network, the students' timeline for their activities, and some relevant metrics associated to them.

The remainder of this paper is organized as follows. Section II presents the different data sources that are aggregate in order to fulfill our research questions. After that, our proposal of the aggregated Learning Analytics dashboard, as a result of the integration of AutoES and the institutional LMS, is detailed in Section III. Section IV describes the visualization of the selected learning parameters, and Section V discusses the implications of this research work and some recommendations are given. Finally, Section V highlights our final remarks and suggests guidelines for future work.

\section{II.DATA SOURCES}

The sources used in this work come from a self-evaluation system, named AutoES (AutoEvaluation System) [21], and the institutional Learning Management System (LMS). From this information, a data aggregation process will be done.

\section{A. AutoES}

The main objective of AutoES [21] is the management of the self-evaluation of practical activities with virtual/remote laboratories and the continuous assessment of the students' progress. It is a service-oriented application, which is considered as the latest generation of Internet-based platforms [20]. Using it, students will be able to perform a selfevaluation of their activities, which they performed with remote laboratories. Additionally, AutoES can solve all the errors made in the activity or configure it completely, with a penalty in the mark for the activity.

AutoES has several main benefits for the members of the learning community, especially within the field of distance higher education. First, it minimizes the response time in correcting students' practical activities, allowing the continuous evaluation process to be performed smoothly. Furthermore, it provides a more detailed monitoring of the students' progress, thereby reducing the time spent on the assessments themselves. The importance of these benefits is really significant, since the number of students enrolled in a course with a distance methodology can become very high. Thus, lecturers can focus on other tasks, such as dynamic adaptation of new activities to students' necessities or expanding the existing ones, which in turn improves the learning process more than devoting their time to correcting the students' activities.

AutoES is made of two different parts: the lecturers 'view and the students' view. From the lecturers' view, lecturers can perform subject management tasks such as selecting the activities for the subject, creating different groups with activities adapted to the students' level, checking students' progress by means of reports, etc. This view is presented by a Web application, named LabManager, which is accessible by lecturers through any Internet browser. For each particular student, Lab Manager provides last, maximum, and mean qualifications for each activity. Lecturers will be able to assign a student's final qualification according to these previous ones. It also includes the groups to which he/she belongs and the corresponding activities assigned. Note that the system allows lecturers to split up the subject's students by levels or types of activities. In addition, the system provides statistics about the student's run status and run time. From the learning process point of view, lecturers have several indicators of the students' performance, among others, number of tries for each activity, number of successful evaluations per activity and student, number of failed evaluations per activity and student, and a summary of the evaluation logs. Finally, a list of recent reports is stored for each of them, which can be checked by lecturers at will.

From the students' view, AutoES can automatically configure and/or evaluate a particular activity. Every time they check an activity, a report is created that summarizes the results of this checking. This report is presented each time the student checks an activity as a console message. So, students find out which parts of a particular activity are wrong and, additionally, AutoES can help them when they are not able to do a part of the activity. All this information will be automatically updated on the server side so that it can be used by lecturers to improve the learning process and to decide on the students' marks.

The architecture of AutoES is shown in Figure 1, including the interaction between its main elements, which are Web Client, Lab Manager, and Web Server.

Apart from the learning indicators that will be detailed in the next section, AutoES offers a set of parameters for each proposed activity and student:

1) Start date and time.

2) Finish date and time.

3) Number of successfully evaluations for that activity.

4) Number of failed evaluations for that activity.

5) Logs of errors.

These parameters are included in the aggregation data and 
in the dashboard.

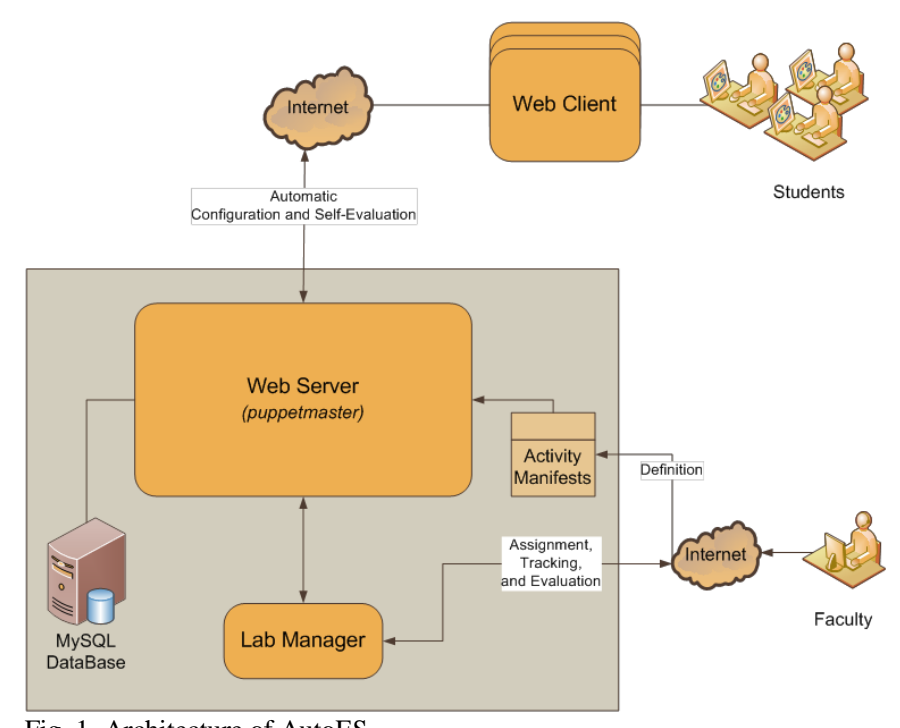

Fig. 1. Architecture of AutoES.

\section{B. The Institutional LMS}

The LA process is focused on all the information gathered from all the activities that are crucial for the lecturer's dailywork, especially when applying to a distance methodology. For this reason, a clear necessity of processing all this information appears in order to allow lecturers to extract interesting conclusions for the dynamic adaptation of the learning process to students. It is clear that the information provided by AutoES allows lecturers to have a partial view of the learning process, and it must be combined with the data contained within the LMS.

Therefore, there is a need to enrich the lecturer knowledge of the learning process through the information gathered by both AutoES and the LMS. After the revision of all the relevant educational tools inside the LMS, the forums have been pointed out as the most relevant information source for collaborative evaluation. The use of asynchronous on-line discussion forums is thought to be essential for the negotiation and exchange of ideas, as well as the development of critical thinking skills, all of which are important components of the collaborative learning process [10], [11], [16]. Furthermore, several studies have demonstrated a high correlation of students' participation levels in discussion forums with positive learning outcomes and knowledge constructions [23], [24].

In this sense, as a result of the integration of both learning environments, the aggregation dashboard can graphically show the students' progress both in an experimental and collaborative way at the same time. Therefore, lecturers can guide each student through the learning process based on his/her particular level of proficiency and grade her/him at the end of the term. In particular, the data aggregation, the computation of learning parameters, and their visualization are detailed below.

\section{Data Aggregation}

As explained before, within the context of the evaluation activities, we have found two learning processes. First, the practical experimentation with the virtual machines picked up by AutoES, and second, the students' knowledge creation through the discussion in the evaluation forums. These learning processes are highlighted by lecturers when they are questioned about how they perform the evaluation of students. Therefore, if we want to represent the learning process into our analysis, at least these two sources of information should be merged: AutoES' events and forums' evaluation messages.

There are other data sources that provide relevant data for the learning process. On one hand, we cannot extract further data from AutoES without changing the basis of the system. Nevertheless, the LMS can offer additional information, such as quizzes scores, activities' deadlines, time spent in the platform, and so on. As this work is a starting point of our research, we only consider the most relevant data sources, but in the future additional data should be aggregated in order to capture all the possible factors. According to this, there are several factors that cannot be obtained neither AutoES nor the LMS, such as personal conditions of students (social environment, health status...), and they may affect the learning process.

Because of the fact that both systems, AutoES and the LMS, have their own data representation, a database merging process is defined. So, in order to have the same representation for both databases, a generic register is created. Afterwards, the data from AutoES and forums are stored in the same database thus further computations are easier.

Each student's interaction is represented by a register within this "merging" process. A register is a structured data generated every time that a student performs an activity which happens at a particular time, and it could produce an output result. Each register contains the following data:

1) A register identifier.

2) The identifier of the user that generates the event.

3) The course to which the students belongs.

4) The type of activity that is represented by the register.

5) An associated report about the activity.

6) The practice associated with the activity.

7) The date and time when the activity takes place.

Thus, a student can produce a set of types of activities inside our learning context, namely:

1) Creation of a user at AutoES (called created event). Students enroll themselves dynamically, thus a register is created in this case. In this case, the report field is empty.

2) When a user starts AutoES tool (called unchanged event). The report field is empty.

3) A successful evaluation that produces a report as an output result (called success event). In this case, the report field contains a brief text that reports about the evaluation.

4) A failed evaluation that produces a report as an output 
result (called fail event). In this case, the report field contains a brief summary about the errors that are found.

5) Publication of a new thread message inside the evaluation forum, where the message is the output result (called init message event). In this case, the report contains the posted message.

6) Response to a previous message inside the evaluation forum, where the message is the result (called response to event). In this case, the report contains the posted message.

7) Initiates a new activity, and the previous activity is finished (called added to event). The report field contains a reference to the finished activity and the activity field contains the identifier of the just started activity.

8) When a user gives up the AutoES tool (called removed event). The report field is empty in this type of register.

For this analysis, not all messages located at the forums are interesting. In this sense, previously to the merging process, the messages have been classified in several topic categories by using the cluster k- means algorithm and a bag-of-words approach. So, messages can be correlated with the evaluation activities due to their content and, additionally, filter which messages are relevant for the learning/evaluation process. Messages not related to the evaluation activities or whose contents are not relevant, are dropped from our study.

\section{DESCRIPTION OF LEARNING INDICATORS}

Lecturers should evaluate not only the results, but also the experimental process conducted in the student's virtual/remote laboratory. During the learning process, it is very relevant to detect students at risk of quitting the activities and help them, because if nothing is done, they will not acquire the required learning skills. So, the main objective of this work is answering the following questions using the aggregation of the information of the previously described data sources.

1. Are the students engaged with the proposed practical activities or are they at risk of quitting the activities?

2. Can we create a system that helps to evaluate if the proposed activities are well-designed?

For that purpose, we have computed three indicators that represent their learning outcomes from the activities and four indicators correlated with the behavior of the student in the forums. All the indicators are graphically represented in the aggregation dashboard so lecturers can easily get an overview of the learning progress of each student.

The "On time" indicator is focused on the time spent on the realization of the evaluation activity. For the whole population, the average time to solve each activity is computed. Each student's time is compared to this average result by computing the student's corresponding z-score. A higher z-score means that the student is delayed with regard to his/her group and he/she is at risk of quitting. As oppose to this, a lower value means that he/she is solving the activities quickly. This indicator is usually higher at the beginning of the course, and it should decrease as the course goes by and the student is achieving the subject's objectives.

In a similar way, the second parameter called "Failure rate", is devoted to analyzing the number of failed evaluations for each student. The number of failed evaluations per activity, the time between failed evaluations, and the student's z-score, calculated by comparing each students' statistics with the average of all the students', are also calculated. A high $\mathrm{z}$-score value means that the student has problems to solve the activity, so the lecturer should offer some additional help. It is also a source of frustration for the student and he/she may decide to quit. A lower value of this indicator means that the student has solved the activity with fewer problems than his/her classmates.

Finally, the third parameter called "Success rate" is correlated to successful evaluations. It is computed similarly to the failed evaluation parameter. The number of success evaluations per activity, the time between success evaluations, and the student's z-score, calculated by comparing each student's statistics with the average of all the students', are also calculated. A high z-score value means that the student has not problems to solve the activity. On the other hand, a low value for "Success rate" indicator in combination with a high value of "Failure rate" indicator could mean that the student is having trouble to solve the activities.

On the other hand, Social Network Analysis (SNA) provides a powerful mechanism for understanding how human relationships are created and developed, as well as detecting communication patterns and structures that should appear from these interactions [13], [26]. In [18], it is proved that there is a correlation between forum interactions and the students' performance. This correlation is also explored by the tool SNAPP [6] and Vercellone-Smith et al. [29] by means of social networks analysis. According to this, a Social Network (SN) can be represented as a directed graph in which nodes are individual or grouped users and links are the relationships among people. Nodes are also used to represent concepts, events, ideas, and other learning elements. These networks are usually built upon gathering and processing the information obtained from the LMS, where interactions among nodes are established in order to acquire new knowledge within a social community.

In our particular case, the creation of a $\mathrm{SN}$ graph for the analysis of educational communities is based on the messages published in discussion forums. More in detail, links between two nodes, where each node represents a particular student, are weighted with the amount of messages exchanged [6]. Thus, the analysis performed of the resulting social network allows lecturers to analyze the interest propagation of their group of students, as observed in Figure 3.

We have computed four basic indicators inside the social network that help lecturers analyze the student's progress and their level of proficiency. 




Fig. 2. Events per User by Including Students' Forums Interactions.

There are a variety of different measures to evaluate the importance, popularity, or social capital of a node within a social network:

1) Degree centrality (interactivity) focuses on individual nodes, it counts the number of edges that a node has. This value represents the interactivity level of the student; that is, how often the student posts in forums. This indicator could have several meanings that are qualified with the rest of learning indicators.

2) Betweenness centrality (broker/hub) of a node is the sum of the fraction of all-pairs shortest paths that pass through that node. Nodes that occur on many shortest paths between other nodes in the graph have a high betweenness centrality score and are more likely to behave as a hub or broker in the network. In this context, students tend to group into communities at forums. The students that behave as hubs or brokers in the social networks allow the exchange of ideas among communities due to the fact that they take part in several of them.

3) Eigenvector centrality (neighborhood) of a node, which is proportional to the sum of the centrality scores of its neighbors. A node is important if it is connected to other important nodes. A node with a small number of influential contacts may outrank one with a larger number of non-popular contacts. Thus, this parameter measures the relevance of the neighbors of a student. A better group of neighbors will help a student to create a better collaborative knowledge and it will encourage him to do the activities.

In addition to popularity measures, we pay attention to the clustering coefficient (integration) for each student. The bachelorhood of a node that represents a student is a set of nodes connected to it by an edge, not including itself inside the social network. The clustering coefficient of a node is the fraction of pairs of its neighbors that have edges between one another. Locally, this indicates how concentrated the neighborhood of a node is. A higher clustering coefficient means that the student has been exchanging messages with a high portion of the classroom.

The combination of these learning indicators allows lecturers to answer our research questions. According to the first question related to the student's engagement with the activities, an interactive student that presents a high degree centrality value with also a high value for "Success rate" indicator and a low value for "Failure rate" indicator means that the student is helping other students with the activities sharing his/her knowledge. It is common that each community is created with at least one student with these parameters. A particular case is if this student could have a high value as betweennes centralities because his/her answers are popular and he/she becomes member of more than one community. And it is also very frequent that the eigenvector centrality value of the student is as high as the clustering coefficient. So, this student is going to successfully pass the activities with high scores.

On the other hand, a very interactive student with a high value for "On time" indicator and a high value for indicator "Failure rate" could have serious problems in order to solve the activity and he/she is searching for help among his/her peers. Lecturers in this case must pay attention to the student and they should offer additional learning resources because he/she is at risk of quitting the activities. Students at risk could also have a low value of betweenness centrality and a low value of eigenvector centrality because he/she is not posting solutions of problems, which are popular messages.

Our second research question is easily answered starting from the previous results. If during the period of an activity, 
the number of students at risk of quitting computed with learning indicators has increased substantially, while during other activities the same students have a more successful performance, maybe lecturers should consider redesign the activity. That activity could be too complex or the resources for the development of the activity are not clear. This type of situation is often accompanied by an increase of the number of exchanged messages at the forums under the label of that activity. So, lecturers must be aware of the evolution of the performance of the students during the course. Further analysis over the interrelation of these parameters could help to detect automatically an activity, whose design is not correct by describing some threshold values. But, lecturers should supervise this classification. In addition, these value thresholds could be correlated to the learning context (subject, course...), thus it is a difficult task for automation.

\section{VISUALIZATION OF LEARNING PARAMETERS}

In order to allow faculty to easily see these indicators and use them to guide the learning/teaching process of students, a dashboard with a Graphical User Interface (GUI) has been developed. Figures 3 and 4 show the most relevant lecturers' interfaces of our proposed LA dashboard.

When a faculty starts browsing in the home view of our LA dashboard, he/she can visualize several graphs, such as the one depicted at Figure 2, which summarize all the events. In addition to offering a global view of what happens in AutoES and evaluation forums at the same time, the lecturer can also observe (as a colored calendar) the set of events generated by each student, as presented in Figure 4(b). This way, a lecturer can easily verify the generated events, and why they are produced. An improvement will be that students and lecturers can compare this activity with the average activity of the course. So, students can be aware of their performance and adapt it in order to improve their learning outcomes.

As we mentioned above, we offer lecturers the possibility of examining the social network generated in the course. The size of students' node is directly proportional to his/her network degree. Additionally, the virtual students' communities represented by the social network are computed by following the Louvain method [2]. Students in the same community are colored with the same color. This visualization is represented at Figure 3.

Finally, we include a graphical visualization for the previously explained indicators; see Figure 4(a). There is a matrix with a cell for each pair <student, indicator>. If a student has a poor performance in an indicator, the cell representing it is colored in a darker red. On the other hand, if the performance of the student for an indicator is good, the indicator cell is colored with a dark blue color. So, lecturers can easily interpret the combined indicators through this graphical representation.

\section{V.DISCUSSION}

This section discusses the implications of this research work by taking into account our current learning context.

\section{A. Learning Context}

In order to focus this work, AutoES will only use activities related to the configuration of network services. Its scope is much broader, since this system has been designed and implemented as a modular system, which is independent of the design and implementation of specific activities with remote laboratories. In this regard, we focus on the "Network Services Management in Operating Systems" (NetServicesOS) course belonging to the "Communications, Networks, and Content Management" post-graduate program at Spanish University for Distance Education (in Spanish, Universidad Nacional de Educación a Distancia - UNED). The duration of the subject is 15 weeks in the first semester of the academic year. The main goals of the NetServicesOS are the deployment and configuration of several network services for Windows and Linux operating systems, such as DNS, DHCP, FTP, Web, etc., using virtual machines (VMs).

Thanks to the use of AutoES, lecturers can track the progress of a large number of students and adapt dynamically the learning/teaching process. Students can also receive timely feedback on their activities - which was totally impossible with our traditional evaluation system based on explanation reports for each activity.

Since the UNED University follows a distance methodology, the main element of interaction among participants in the learning/teaching process (students and lecturers) are forums, which motivate the learning/teaching process of the subject and allow the formation of virtual social communities. Lecturers play a vital role in promoting a suitable learning space that motivates the interaction among students. In our particular case, lecturers provide students with a set of practical activities which require a great interaction among students to solve them. Lecturers have created a dedicated forum related to the activities for these purposes. The interactions in forums are also taken into account by lecturers when calculating students' final grades.



Fig. 3. Representation of the Social Network into the Dashboard. 


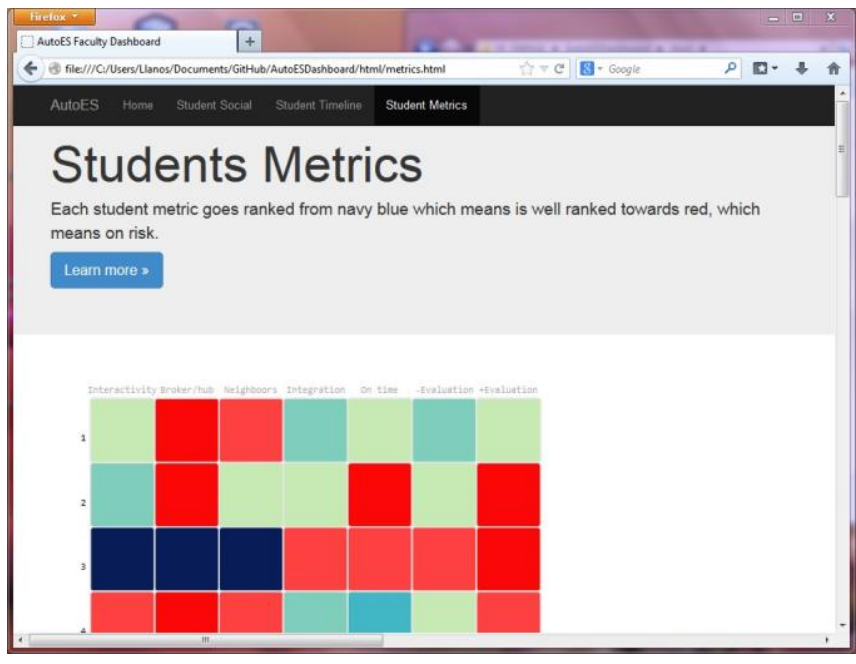

(a) Table Summary of the Student's Metrics in the Dashboard.

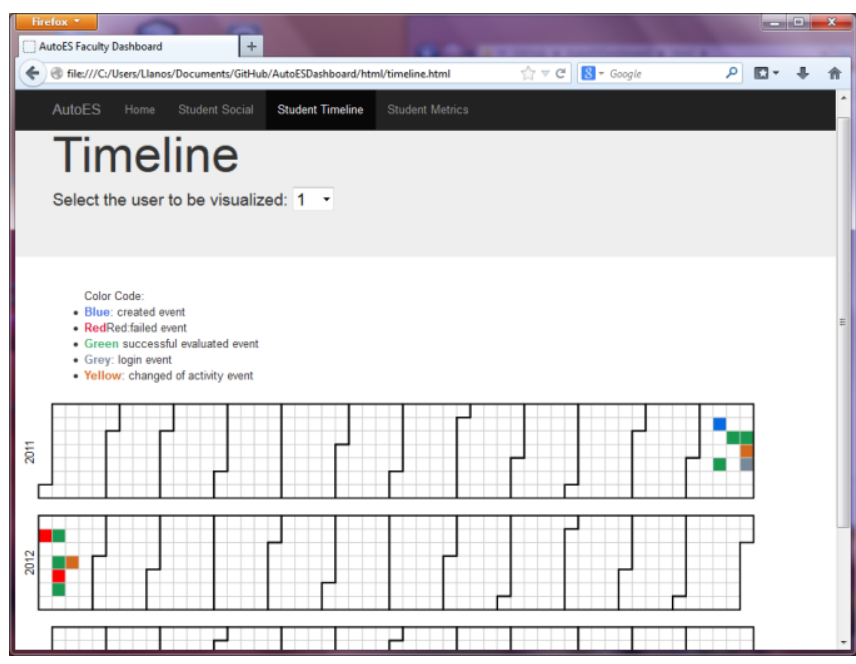

(b) Calendar Interface by Representing Each Student's Event.

Fig. 4. Lecturers' Visualizations in our Proposed LA dashboard (Metrics and Calendar).

\section{B. Results}

After the cleaning phase previous to the data aggregation phase, an $83 \%$ percentage of messages are relevant to the analysis. The discarded messages are not related to the subject development. Instead, they are Christmas greetings, the place where students can buy/find the bibliography, or students introductions. These topics have a very low correlation with any subject topic, but they are very correlated to an external event. They are initially inactive, although they become very active within a particular time sub-window. After that, they become again inactive. Topic characterization and its impact in the learning outcomes have been widely studied at several works, such as [7], [29], and [30].

As a result of this merging process, there are 2179 events located in the final database, where 1583 are forum's events; this result is depicted in Figure 2. As stated above, there is a high percentage of information related to the student's learning process within the LMS. Therefore, it can be seen that the aggregation of the LMS data provides a large amount of information of great interest in order to guide our learning/teaching process. An initial approach of the statistical analysis of these events shows some relevant results, which are reflected in Figure 2. Each practice takes eleven days to be completed by a student in average.

If we pay attention to the correlation of messages and evaluation activities, we have found that most of the failed evaluations are followed by a message event; almost the $73 \%$ (see Figure 5). The visualization of Figure 5 shows the number of events per day. Each circle represents the amount of events. Thus, as the number of events is bigger, the circle is redder and its size is bigger. As we can see at day 4, as example, after the first occurrence of Failed events (which means that students fails the evaluation test), the number of events of "New threads" (which means a student has initiated a new thread in the forum) is increased. The following days the number of events of type "Responses", which mean that students are replying messages at the forums, is higher. This frequency analysis has been completed with a topic detection analysis in order to correlate forum messages and failure events.

Also, at least the $80 \%$ of students have posted a correlated message when they are moving from one activity to other. And $68 \%$ students who did not use AutoES have replied to the doubts of the AutoES students. This means that the doubts are more related to the development of the experiment itself than to the use of AutoES.

From the Social Network graph, depicted at Figure 3, three communities are detected: blue nodes, green nodes and orange nodes. Most of the students belong to the blue cluster, while the other two are smaller. Each node represents a student, and an arc between two students represents a message exchange. The size of the representation of the node indicates the popularity of the student. There is a clear big blue node in the center of the social network that is the lecturer, who plays a relevant role in the bigger community. In the same figure, we can clearly see that there are several students which have exchanged a very few messages. These students could be at risk and the lecturer should pay attention to the other learning indicators.

There are 36 students in the classroom who decided to work with AutoES. It is also relevant that students execute more than once the evaluation of each practice if he/she has obtained a successful evaluation. This fact can be easily detected with the timeline representation (see Figure 4(b)). As an example, the first activity, when a user is successfully evaluated, he/she is evaluated nine times in average. This situation occurs more often at the beginning of the use of AutoES rather than in the last part. So, students need a period of time for learn how to use the AutoES tool. Also, AutoES output must be improved in order to help students with this 




Fig. 5. Graph related to the Evolution of the Activity during the Course.

regard. Another relevant result is that failed evaluations are more common at the first activities rather than the last ones. While the average of failed evaluations of first practice is three by student; the average of failed evaluations of the last activity is 0.47 .

There is high percentage of quitting the platform. At least 12 students stopped using the platform at the end of the course. Half of them have quitted the platform during the first activity. This fact is reflected at the dashboard Student Metrics (see Figure 4(a)), such as the learning indicators of the student 3 at Figure 4(a). This student has red values in the "On time", "Successful rate", "Failure rate" and clustering coefficient indicators. On the other hand, he has blue values degree centrality, betweenness centrality and eigenvector centrality. This student belongs to the orange community detected in the social network. It seems that he/she has sought for advice at the forum but he/she could not solve the activity. Finally, this student has stopped using AutoES.

On the other hand, at the same Figure 4(a), although student 1 has a red colored value for betweenness centrality and a pale value for eigenvector centrality, he/she has light green values for the rest of the learning indicators, which means he/she is successfully completing the activities with the help of forums. In fact, this student achieved a high ranking at the course

The activity of students at risk is in some cases average, with pale red values for "On time", "Successful rate" and "Failure rate" indicators, although most of them have strong red values. Out of the 12 students who quitted using AutoES, three of them, as the student 3 of our example, have blue values for degree centrality, betweenness centrality and eigenvector centrality. This means that students were searching for advice at the forums before quitting the activity. The rest of them also have pale red values for the seven learning indicators. Lecturers must detect these students and should offer them additional help to prevent their desertion from the platform.

The number of students that have stopped using the tool is around $30 \%$ of the total. On this fact, students were requested to fill in a survey about the tool. According to this, most of the students found AutoES useful and easy to use, as detailed in
[21]. From the obtained feedback, the main drawback of AutoES was that students were not confident with the automated evaluation of the tool. This topic has also arisen in the forum messages. Thus, the activities design seems correct. But, it looks like the supporting documentation must be increased.

The obtained results from the research questions presented in this section have been validated with the real lecturers during the courses. There is a correlation among the score of the activities and the information obtained from the proposed system.

The proposed dashboard is useful with this regard. Moreover, students should have a reporting tool, such as a dashboard, that allows them to keep track of their learning process. Lecturers should periodically supervise the results of this dashboard in terms of the design of the activities.

\section{CONCLUSIONS AND FUTURE WORK}

This work integrates the information gathered from AutoES, a Learning Analytics (LA) system, and the most relevant tools of our institutional LMS. Therefore, lecturers are able to acquire new useful knowledge in order to improve the learning/teaching process of our subjects. As an example, a subject focused on the configuration of network services has been chosen to implement our approach. In particular, a graphical dashboard has been built from this integration and a set of learning parameters has been analyzed, so that lecturers can guide each student through the learning process based on his/her particular knowledge-level and grade her/him at the end of the term.

As a future work, we plan to improve the functionality of the system by developing alternative indicators for the analysis of the aggregated data from AutoES and forums' messages, this way improving the adaptation of the evaluation resources to achieve more intelligent curricula [17]. Additionally, we will also aggregate other information sources that can improve the vision of the learning process. Finally, different frameworks or contexts from EHEA, as the ones proposed by the ASEE Educational Research Methods (ERM) Division [1], could be explored in order to analyze if the results obtained 
are similar and/or there is a need of making some changes.

\section{ACKNOWLEGDEMENTS}

Authors would like to acknowledge the support of the following European Union projects: RIPLECS (517836- LLP1-2011-1-ES-ERASMUS-ESMO), PAC (517742-LLP-12011-1-BG-ERASMUS-ECUE), EMTM (2011-1-PL1LEO05-19883), and MUREE (530332-TEMPUS-1-2012-1JO-TEMPUS-JPCR). Furthermore, we also thank the Community of Madrid for the support of E-Madrid Network of Excellence (S2009/TIC-1650).

\section{REFERENCES}

[1] American Society for Engineering Education Educational Research and Methods Division. Web page at http://erm.asee.org/. Date of last access: June 25, 2014.

[2] V. Blondel, J. Guillaume, R. Lambiotte, and E. Mech. Fast unfolding of communities in large networks. J. Stat. Mech, 2008.

[3] P. Brusilovsky and E. Millán. User models for adaptive hypermedia and adaptive educational systems. In P. Brusilovsky, A. Kobsa, and W. Nejdl, editors, The Adaptive Web: Methods and Strategies of Web Personalization, Lecture Notes in Computer Science, pages 3-53. Springer Berlin Heidelberg, 2007.

[4] R. M. Carro, A. Ortigosa, E. Martín, and J. H. Schlichter. Dynamic generation of adaptive web-based collaborative courses. In CRIWG, pages 191-198, 2003.

[5] T. N. M. Consortium. The horizon report (2011 edition). On-Line, 2011. Date of last access: June 25, 2014.

[6] S. Dawson, L. Lockyer, A. Bakharia, E. Heathcote, L. Macfadyen, P. Long, R. Phillips, and P. Poronnik. SNAPP. On-Line, 2011. Date of last access: June 25, 2014.

[7] L. P. Dringus and T. Ellis. Temporal transitions in participation flow in an asynchronous discussion forum. Computers and Education, 54(2):340-349, 2010.

[8] T. Elias. Learning analytics: Definitions, processes and potential. OnLine, 2011. Date of last access: June 25, 2014.

[9] N. Furugori, H. Sato, H. Ogata, Y. Ochi, and Y. Yano. COALE: Collaborative and adaptive learning environment. In Proceedings of CSCL 2002, pages 493-494, 2002.

[10] D. R. Garrison, T. Anderson, and W. Archer. Critical thinking, cognitive presence, and computer conferencing in distance education. American Journal of Distance Education, 15(1):7-23, 2001

[11] D. R. Garrison and M. Cleveland-Innes. Facilitating cognitive presence in online learning: Interaction is not enough. American Journal of Distance Education, 19(3):133-148, 2005.

[12] E. Gaudioso and J. Boticario. Supporting personalization in virtual communities in distance education. World Scientific Publishing Company, 2002.

[13] R. Hanneman and M. Riddle. Introduction to social network methods (online textbook). On-Line, 2005. Date of last access: June 25, 2014.

[14] I.-H. Hsiao and P. Brusilovsky. The role of community feedback in the student example authoring process: An evaluation of annotex. British Journal of Educational Technology, 42(3):482-499, 2011.

[15] A. Kobsa, J. Koenemann, and W. Pohl. Personalized hypermedia presentation techniques for improving online customer relationships. The Knowledge Engineering Review, 16:111-155, 2001.

[16] J. B. Pena-Shaff and C. Nicholls. Analyzing student interactions and meaning construction in computer bulletin board discussions. Computers and Education, 42(3):243-265, 2004.

[17] A. Robles-Gómez, S. Ros, R. Hernández, L. Tobarra, A. C. Caminero, R. Pastor, M. Rodríguez-Artacho, M. Castro, E. SanCristóbal, and M. Tawfik. Towards an adaptive system for the evaluation of network services. In 2013 Frontiers in Education Conference - Energizing the Future, 2013. FIE'13. 43rd Annual, pages 1-7. IEEE, 2013.
[18] C. Romero, M. I. López, J. M. Luna, and S. Ventura. Predicting students' final performance from participation in on-line discussion forums. Computers and Education, 68:458-472, 2013.

[19] Pardo, D. Burgos, and C. D. Kloos. Monitoring student progress using virtual appliances: A case study. Computers and Education, 58(4):1058-1067, 2012.

[20] S. Ros, R. Hernández, A. Robles-Gomez, A. C. Caminero, Ll. Tobarra, and E. SanCristobal. Open service-oriented platforms for personal learning environments. IEEE Internet Computing, 17(4):26-31, 2013.

[21] S. Ros, A. Robles-Gómez, R. Hernández, A. Caminero, and R. Pastor. Using virtualization and automatic evaluation: Adapting network services management courses to the EHEA. IEEE Transactions on Education, 55(2):196-202, 2012.

[22] C. Saul and H.-D. Wuttke. Towards a high-level integration of interactive tools with e-assessments. In Proceeding of IEEE International Conference on Advanced Learning Technologies (ICALT), 2012.

[23] T. Schellens and M. Valcke. Fostering knowledge construction in university students through asynchronous discussion groups. Computer and Education, 46(4):349-370, 2006.

[24] T. Schellens, H. van Keer, M. Valcke, and B. de Wever. Learning in asynchronous discussion groups: a multilevel approach to study the influence of student, group and task characteristics. Behavior and Information Technology, 26(1):55-71, 2007.

[25] L. Schrum, M. D. Burbank, and R. Capps. Preparing future teachers for diverse schools in an online learning community: Perceptions and practice. The Internet and Higher Education, 10(3):204-211, 2007.

[26] J. P. Scott. Social Network Analysis: A Handbook. SAGE Publications, 2000

[27] Spanish Government. Ministry of Education, Culture and Sports. What is bologna? (In Spanish). On-Line, 2009. Date of last access: June 25, 2014.

[28] Ll. Tobarra, A. Robles-Gómez, S. Ros, R. Hernández, and A. C. Caminero. Analyzing the students' behavior and relevant topics in virtual learning communities. Computers in Human Behavior, 31(2):659-669, 2014.

[29] P. Vercellone-Smith, K. Jablokow, and C. Friede. Characterizing communication networks in a web-based classroom: Cognitive styles and linguistic behavior of self-organizing groups in online discussions. Computers and Education, 59(2):222-235, 2012.

[30] E. Webb, A. Jones, P. Barker, and P. van Schaik. Using e-learning dialogues in higher education. Innovations in Education and Teaching International, 41(1): 93-103, 2004.

[31] G. Weber and P. Brusilovsky. ELM-ART: An adaptive versatile system for web-based instruction. International Journal of Artificial Intelligence in Education, 12:351-384, 2001.

Llanos Tobarra received her M.Sc. degree in Computer Science in 2004 and his Ph.D. in Computer Science in 2009, both from the University of CastillaLa Mancha, Albacete, Spain. She is currently a Lecturer at the Control and Communication Systems Department at the Spanish University for Distance Education, UNED. Her interests include security support in distributed systems and the analysis of social networks and remote labs for e-learning. She has co-authored more than 30 publications in international journals and conferences on these topics.

Salvador Ros (SM'07) received his M.Sc. degree in Physics, specialized in Control and Automatic Systems, in 1991 at Complutense University, Madrid, Spain, and his Ph.D. in Computer Science in 2012 at the Spanish University for Distance Education, UNED. He is Associate Professor at the Control and Communication Systems Department at UNED. For five years (May 2004June 2009), he has been an Educational Technologies Manager at UNED. He managed the Virtual Campus and Multimedia Production at UNED. He has also been a Technological Projects Evaluator for the Scientific Investigation, Development and Technological Innovation, Spanish National Program. He is currently a Vice Dean of Technologies at School of Computer Science at UNED. He is a senior member of IEEE.

Roberto Hernández (SM'07) received his M.Sc. degree in Physics, specialized in Electronics, in 1989 at Complutense University, Madrid, Spain. He also received his Ph.D. in Sciences in 1994 at Spanish University for Distance Education, UNED, and Madrid, Spain. He is Associate Professor at 
the Control and Communication Systems Department at UNED. He was the Dean of Technologies at School of Computer Science at UNED from 2005 to 2013. His research interests include quality of service support in distributed systems and development of infrastructures for e-learning. He has co-authored more than 60 publications in international journals and conferences on these topics. He is a senior member of IEEE.

Antonio Robles-Gómez (M'10) received his M.Sc. degree in Computer Science in 2004 and his Ph.D. in Computer Science in 2008, both from the University of Castilla-La Mancha, Albacete, Spain. He is an Assistant Professor at the Control and Communication Systems Department at the Spanish University for Distance Education, UNED. He teaches graduate and postgraduate courses related to the network interconnection and security domains. His research interests include quality of service support in distributed systems and development of infrastructures for e-learning. He has co-authored more than 35 publications in international journals and conferences on these topics. He is a member of IEEE.

Agustín C. Caminero (M'10) received his M.Sc. and Ph.D. degrees in Computer Science in 2004 and 2009 respectively, both from the University of Castilla-La Mancha, Albacete, Spain. After that, he was awarded with a Postdoctoral grant at Complutense University, Madrid, Spain. He is an Assistant Professor at the Control and Communication Systems Department at the Spanish University for Distance Education, UNED. His interests include quality of service support in parallel distributed computing systems, and development of infrastructures for e-learning. He has co-authored more than 35 publications in international journals and conferences on these topics. $\mathrm{He}$ is a member of IEEE.

Rafael Pastor (M'06) received his M.Sc. degree in Physics in 1994 at Complutense University, Madrid, Spain. He also received his Ph.D in 2006 at Spanish University for Distance Education, UNED, Madrid, Spain. He is an Associate Professor at the Control and Communication Systems Department at UNED. From 1994 to 2009, he worked at the UNED Computer Sciences Faculty, and as Innovation Manager of the Innovation and Development Centre of UNED. Since then he has been a General Manager, adding innovative services in the learning model of UNED. He is a member of the IEEE, Spanish Education Society, and .LRN Board Consortium. 